Article

\title{
Effect of Multiplicity Fluctuation in Cobalt Ions on Crystal Structure, Magnetic and Electrical Properties of $\mathrm{NdCoO}_{3}$ and $\mathrm{SmCoO}_{3}$
}

Vyacheslav A. Dudnikov ${ }^{1}\left(\mathbb{D}\right.$, , Yuri S. Orlov ${ }^{1,2, *}$, Leonid A. Solovyov ${ }^{3}$, Sergey N. Vereshchagin ${ }^{3}{ }^{(1)}$, Sergey Yu. Gavrilkin ${ }^{4}$, Alexey Yu. Tsvetkov ${ }^{4}$, Dmitriy A. Velikanov ${ }^{1}$, Michael V. Gorev ${ }^{1,2}{ }^{\mathbb{D}}$, Sergey V. Novikov ${ }^{5}$ and Sergey G. Ovchinnikov ${ }^{1,2}$ (i)

1 Kirensky Institute of Physics, Federal Research Center KSC SB RAS, 660036 Krasnoyarsk, Russia; slad63@yandex.ru (V.A.D.); velikanov@iph.krasn.ru (D.A.V.); gorev@iph.krasn.ru (M.V.G); sgo@iph.krasn.ru (S.G.O.)

2 Institute of Engineering Physics and Radio Electronics, Siberian Federal University, 660041 Krasnoyarsk, Russia

3 Institute of Chemistry and Chemical Technology, Federal Research Center KSC SB RAS, 660036 Krasnoyarsk, Russia; leosol@icct.ru (L.A.S.); snv@icct.ru (S.N.V.)

4 Lebedev Physical Institute of the Russian Academy of Sciences, 119991, Moscow, Russia; gavrs@lebedev.ru (S.Y.G.); tsvetkov@lebedev.ru (A.Y.T.)

5 Ioffe Institute of the Russian Academy of Sciences, 194021 St. Petersburg, Russia; snpochta@gmail.com

* Correspondence: jso.krasn@mail.ru

Received: 29 January 2020; Accepted: 9 March 2020; Published: 12 March 2020

\begin{abstract}
The structural, magnetic, electrical, and dilatation properties of the rare-earth $\mathrm{NdCoO}_{3}$ and $\mathrm{SmCoO}_{3}$ cobaltites were investigated. Their comparative analysis was carried out and the effect of multiplicity fluctuations on physical properties of the studied cobaltites was considered. Correlations between the spin state change of cobalt ions and the temperature dependence anomalies of the lattice parameters, magnetic susceptibility, volume thermal expansion coefficient, and electrical resistance have been revealed. A comparison of the results with well-studied $\mathrm{GdCoO}_{3}$ allows one to single out both the general tendencies inherent in all rare-earth cobaltites taking into account the lanthanide contraction and peculiar properties of the samples containing $\mathrm{Nd}$ and $\mathrm{Sm}$.
\end{abstract}

Keywords: rare-earth cobalt oxides; multiplicity fluctuations; structural; magnetic; electrical; and dilatation properties

\section{Introduction}

The unusual temperature dependence of the magnetic susceptibility and transport properties of $\mathrm{LaCoO}_{3}$ cobalt oxide [1-4] have led to the active study of $\mathrm{RCoO}_{3}$ cobaltites ( $\mathrm{R}$ is a rare-earth element, $\mathrm{RE}$ ) and their derivatives for half a century. A characteristic property of such compounds is their proximity to the spin crossover or the approximate equality of Hund's energy $J_{H}$ and the $10 D q$ crystal field in the $\mathrm{CoO}_{6}$ octahedral complexes formed in a rhombohedral or rhombical distorted perovskite-like structure [5-7]. This leads to multiplicity fluctuations or thermal fluctuations of the spin value (we want to emphasize that the spin value fluctuations considered here should not be understood as spin fluctuations. The latter usually means fluctuations of the spin projection. Many years ago, Vonsovskii introduced a term «multiplicity fluctuations» to discuss a variation of the magnitude of the spin in the $d$ shell [8]) and competition between the low-spin (LS, $\mathrm{S}=0, \mathrm{t}_{2 \mathrm{~g}}{ }^{6}$ ) and high-spin (HS, $\mathrm{S}=2, \mathrm{t}_{2 \mathrm{~g}}{ }^{4} \mathrm{eg}_{\mathrm{g}}{ }^{2}$ ) states of the $\mathrm{Co}^{3+}$ ion, with their intensity depending on the RE ionic radius ("lanthanide contraction") and environmental factors such as temperature or pressure. It is this competition, combined with the 
original properties of the rare-earths by themselves, that causes the unique physical properties of the rare-earth cobalt oxides.

Usually the spin crossover is related to the different multiplets level crossing, under high pressure in many iron oxides it is rather abrupt at some critical pressure $P_{C}$ with the width of crossover dependent on temperature, for the $\mathrm{Fe}_{x} \mathrm{Mg}_{1-\mathrm{x}} \mathrm{O}$ measurements at $5 \mathrm{~K}$ found the width close to zero [9]. Near $P_{C}$ the spin gap defined as the HS and LS energy difference may be comparable to the thermal energy $k_{B} T$ and the spin crossover can be revealed in the temperature dependences of various material properties. It is the case of $\mathrm{LaCoO}_{3}$ and other cobaltites. Rare-earth cobaltites in contrast to iron oxides turn out to be in the LS- state already at $T=0$ and at zero applied pressure; i.e., the spin crossover already occurs in the course of the formation of their structure owing to the "chemical pressure» determining the equilibrium volume of the unit cell.

The crystal field $10 D q$, in contrast to the intraionic exchange interaction $J_{H}$, can vary depending on the interatomic metal-ligand distance. Hydrostatic or chemical pressure and stretching make it possible to influence the strength of the crystal field and, thus, the population of $e_{g}$ and $t_{2 g}$ orbitals. In [10] authors reported an unambiguous demonstration of dimensionality control of $d$-orbital occupation with different symmetries $\left(t_{2 g}\right.$ and $\left.e_{g}\right)$ in atomically thin Mott insulator-band insulator oxide superlattices. The heating induced lattice expansion also vary the crystal field value and related smooth spin crossover effects are the subject of our paper.

The most studied cobaltite compound is $\mathrm{LaCoO}_{3}[11,12]$. The ground state of cobalt ions is defined as the nonmagnetic LS- state without any doubt. A series of EPR experiments [13] and X-ray spectroscopy data [14] on $\mathrm{LaCoO}_{3}$ compositions indicate the cobalt ions transition from the low-spin state to the high-spin state with increasing temperature. This confirms the scheme of multi-electron level given in [15], where the ground LS- state is separated by the spin gap $\Delta_{S}$ from the HS- state splitted into sublevels with the total effective momentum $\widetilde{J}=1,2,3$ due to the spin - orbit interaction. This scheme is consistent with the Tanabe - Sugano diagrams for transition metal ions in octahedral complexes developed in perovskite-like materials [16]. The approximate equality of the Curie constant at room temperature to the value given by $S=1$ mimics the presence of the intermediate-spin (IS, $\mathrm{S}=1, \mathrm{t}_{2 \mathrm{~g}}{ }^{5} \mathrm{eg}^{1}$ ) states, but it follows from the smooth temperature dependence of the average magnetic moment of the mixed LS- and HS- states [17]. Multielectron calculations of the LDA+GTB electronic structure and the properties of $\mathrm{LaCoO}_{3}$ [17] using the term scheme [15] have shown that, due to multiplicity fluctuations, the effective spin is determined by a combination of LS- and HS- states and depends on temperature, therefore it is close to 1 near room temperature and the saturation $S=2$ has been reached only at high temperatures up to 1000K.

The properties of other rare-earth cobaltites have been studiedless. However, the similar features both in magnetic susceptibility behavior $\chi(T)$, thermal dilatation $\alpha(T)$, heat capacity $C_{p}(T)$, electrical resistance $\rho(T)$, have been observed for some $\mathrm{RCoO}_{3}$ compounds similar to the known for $\mathrm{LaCoO}_{3}$ [18-25]. With increasing the RE atomic number, the unit cell volume decreases, the additional chemical pressure increases, and the spin gap $\Delta_{\mathrm{S}}$ increases, stabilizing the LS- state of cobalt ions to higher temperatures [26]. Moreover, the anomalies in the behavior of $\chi(T), \alpha(T), C_{p}(T)$, and $\rho(T)$ shift to the region of higher temperatures and are smoother, giving a correlation between electromagnetic and thermodynamic properties. It should be noted that in most cases the magnetic transition temperatures of the $\mathrm{ReCoO}_{3}$ compounds associated with the magnetic moment ordering of $4 f$ - ions are low, $T_{N} \sim 1$ $\mathrm{K}$ [27]. In the paramagnetic region, large RE moments make the main contribution to the magnetic susceptibility; however, a small $\mathrm{Co}^{3+}$ ions contribution can be distinguished only at high temperatures. This is probably the reason that most of the experimental work is devoted to the study of the thermodynamic and transport properties of cobaltites $[5,7,28,29]$. $\mathrm{LaCoO}_{3}$ is an exception, when total magnetic susceptibility is due to $\mathrm{Co}^{3+}$ ions. The magnetic properties of other rare-earth cobaltites have less been studied [30-33]. 
Considering the potential for extensive use of perovskite-like complex cobalt oxides as solid state oxide power sources [34-36], oxygen membranes [37], gas sensors [38], etc., a more detailed study of the rare-earth cobaltite properties seems to be appropriate.

In current paper structural, magnetic, electrical, and dilatation properties of the rare-earth cobaltites $\mathrm{NdCoO}_{3}$ and $\mathrm{SmCoO}_{3}$ have been studied and their comparative analysis was carried out. The influence of the multiplicity fluctuations of $\mathrm{Co}^{3+}$ ions on physical properties of the studied cobaltites is considered within a virtual crystal model. In this model previously proposed in the study of $\mathrm{GdCoO}_{3}$ the average crystal volume at temperature $T$ is determined by the volume superposition in LS- иHS- states with statistical weights given by the population of LS- and HS- terms [39]. Then, the maximum of anomalous thermal dilatation for $\mathrm{GdCoO}_{3}$ was found at the temperature of about $750 \mathrm{~K}$ in [5]. A lower lanthanide compression for $\mathrm{Nd}^{3+}$ and $\mathrm{Sm}^{3+}$ ions as compared to $\mathrm{Gd}^{3+}$ can be expected to enhance the effects of multiplicity fluctuations in a more convenient temperature range from 200 to $700 \mathrm{~K}$ to measure.

\section{Samples and Experimental Methods}

The rare-earth cobaltites $\mathrm{NdCoO}_{3}$ and $\mathrm{SmCoO}_{3}$ were obtained by conventional ceramic processing using a stoichiometric amount of oxides $\mathrm{Co}_{3} \mathrm{O}_{4}, 99.7 \%$ (metals basis, Sigma-Aldrich, St. Louis, $\mathrm{MO}$, USA), $\mathrm{Nd}_{2} \mathrm{O}_{3}$ and $\mathrm{Sm}_{2} \mathrm{O}_{3}, 99.99 \%$ (Rare Metals Plant, Novosibirsk, Russia), which were thoroughly mixed and the resulting mixture was annealed at a temperature of $120^{\circ} \mathrm{C}$ for $36 \mathrm{~h}$ with intermediate grinding. After annealing, the mixture was ground again, tablets were pressed in bars of $5 \mathrm{~mm} \times$ $13 \mathrm{~mm} \times 1 \mathrm{~mm}$, which were then annealed in air at a temperature of $1200^{\circ} \mathrm{C}$ for $8 \mathrm{~h}$ and cooled together with the furnace up to room temperature at $2{ }^{\circ} \mathrm{C} / \mathrm{min}$.

Powder X-ray diffraction (PXRD) data were collected on a PANalyticalX'Pert PRO powder diffractometer (Eindhoven, Netherlands) equipped with a solid- state detector using the $\mathrm{CoK}_{\alpha}$-radiation in the range of $2 \theta=20-130^{\circ}$ and within $300 \mathrm{~K}$ to $1000 \mathrm{~K}$. The $\mathrm{RCoO}_{3}$ samples $(\mathrm{R}=\mathrm{Nd}, \mathrm{Sm})$ were ground in octane in an agate mortar, dried and placed in a flat holder for PXRD measurements in the Bragg-Brentano geometry. X-ray investigations at high temperature were carried out in an Anton Paar HTK $1200 \mathrm{~N}$ (AntonPaar, Austria) high-temperature chamber with sample rotation and self-adjustment. The samples were preliminarily kept in a high-temperature chamber for $2 \mathrm{~h}$ at a temperature of 1000K. The crystal lattice parameters were refined using the derivative difference minimization method (DDM) [40].

Static magnetization measurements in the temperature range from 1.8 to $400 \mathrm{~K}$ and magnetic field up to 50000 Oe were carried out with a MPMS-XL Quantum Design SQUID magnetometer (USA).

Thermal expansion was studied in the temperature range 100-700 K with a Netzsch DIL-402C induction dilatometer in dynamic mode with heating and cooling rates of $3 \mathrm{~K} / \mathrm{min}$ when purged with dry helium $(\mathrm{O} 2$ content $\approx 0.05 \%$ of the volume). The rod load on the sample is $30 \mathrm{sN}$. The fused silica standards to calibrate and account for the dilatation of measuring system were used.

The temperature dependences of the electrical resistance were obtained with the universal installation-Physical Properties Measurement System (PPMS-9) Quantum Design (USA) at the core facilities center of Lebedev Physical Institute of the Russian Academy of Sciences (Moscow).

\section{Results and Discussions}

\subsection{X-Ray Phase and X-Ray Diffraction Analysis}

According to the PXRD analysis, the amount of cobalt oxide impurity in the samples was $1.5 \%$ and $2 \%$ for $\mathrm{SmCoO}_{3}$ and $\mathrm{NdCoO}_{3}$, respectively. Within the whole temperature range studied, the main phases have an orthorhombic perovskite-type structure with the Pbnm- space group. The experimental, calculated, and difference PXRD profiles after the DDM refinement at $300 \mathrm{~K}$ and $1000 \mathrm{~K}$ are presented in Figure 1. 

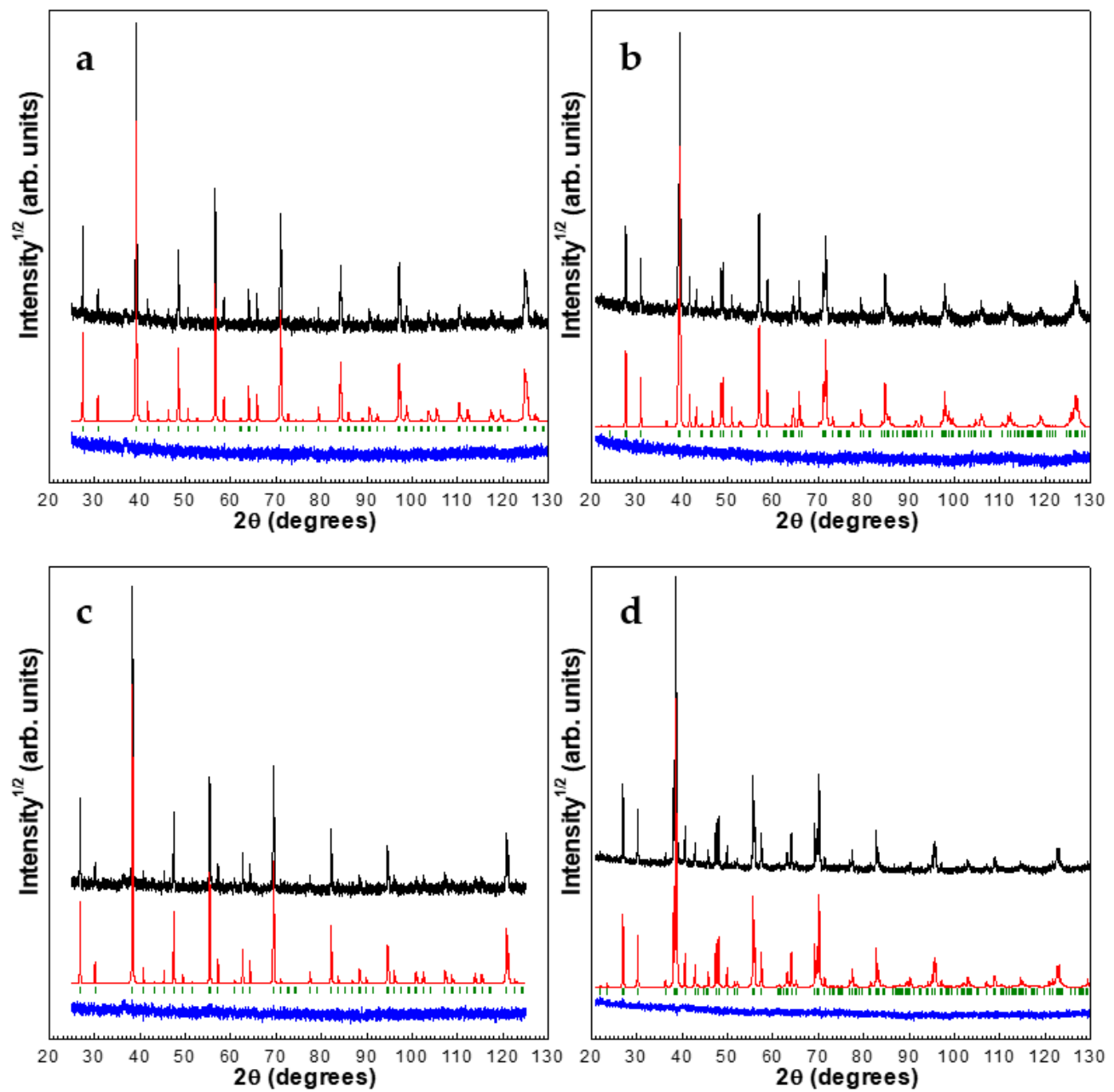

Figure 1. Experimental (upper, black), calculated (middle, red) and difference (lower, blue) Powder $X$-ray diffraction (PXRD) profiles after derivative difference minimization method (DDM) refinement; (a) $\mathrm{NdCoO}_{3}$ at $300 \mathrm{~K}$, (b) $\mathrm{SmCoO}_{3}$ at $300 \mathrm{~K}$, (c) $\mathrm{NdCoO}_{3}$ at $1000 \mathrm{~K}$, and (d) $\mathrm{SmCoO}_{3}$ at $1000 \mathrm{~K}$.

The room temperature structural characteristics are consistent with other data available [41,42]. The crystal lattice parameters at various temperatures are summarized in Table 1 . The deviation of the oxygen nonstoichiometry index from $\delta=3$, according to thermogravimetric analysis, does not exceed $0.6 \%$.

Table 1. Crystal lattice parameters for $\mathrm{NdCoO}_{3}$ and $\mathrm{SmCoO}_{3}$ at various temperatures.

\begin{tabular}{|c|c|c|c|c|c|c|c|c|}
\hline \multirow[b]{2}{*}{$\mathrm{T}, \mathrm{K}$} & \multicolumn{4}{|c|}{$\mathrm{NdCoO}_{3}$} & \multicolumn{4}{|c|}{$\mathrm{SmCoO}_{3}$} \\
\hline & $a, \AA$ & $b, \AA$ & $c, \AA$ & $\mathrm{V}, \AA^{3}$ & $a, \AA$ & $b, \AA$ & $c, \AA$ & $\mathrm{V}, \AA^{3}$ \\
\hline 300 & $5.3478(1)$ & $5.3324(1)$ & $7.5505(2)$ & 215.32(1) & $5.2887(1)$ & $5.3517(3)$ & $7.5031(2)$ & $212.37(1)$ \\
\hline 400 & $5.3591(1)$ & $5.3463(2)$ & $7.5677(2)$ & $216.82(1)$ & $5.2961(1)$ & $5.3572(1)$ & $7.5130(2)$ & $213.16(1)$ \\
\hline 500 & $5.3733(1)$ & $5.3649(1)$ & $7.5909(3)$ & $218.82(1)$ & $5.3082(1)$ & $5.3726(1)$ & 7.5291(1) & $214.72(1)$ \\
\hline 600 & $5.3907(2)$ & $5.3888(1)$ & $7.6176(2)$ & $221.29(1)$ & $5.3246(1)$ & $5.3985(1)$ & $7.5521(1)$ & $217.08(1)$ \\
\hline 700 & $5.4067(1)$ & $5.4106(1)$ & $7.6427(3)$ & $223.57(1)$ & $5.3423(1)$ & $5.4292(1)$ & $7.5777(1)$ & $219.78(1)$ \\
\hline 800 & $5.4208(1)$ & $5.4270(1)$ & $7.6635(1)$ & $225.45(1)$ & $5.3573(1)$ & $5.4519(1)$ & 7.6001(1) & 221.98(1) \\
\hline 900 & $5.4332(1)$ & $5.4404(1)$ & $7.6819(2)$ & $227.07(1)$ & $5.3707(1)$ & $5.4683(1)$ & 7.6197(1) & $223.78(1)$ \\
\hline 1000 & $5.4451(1)$ & $5.4521(2)$ & $7.6989(3)$ & $228.56(1)$ & $5.3831(1)$ & $5.4816(1)$ & $7.6380(1)$ & $225.38(1)$ \\
\hline
\end{tabular}


The temperature dependences of volume expansion coefficient for $\mathrm{NdCoO}_{3}, \mathrm{SmCoO}_{3}$, and $\mathrm{GdCoO}_{3}$ arepresented in Figure 2.

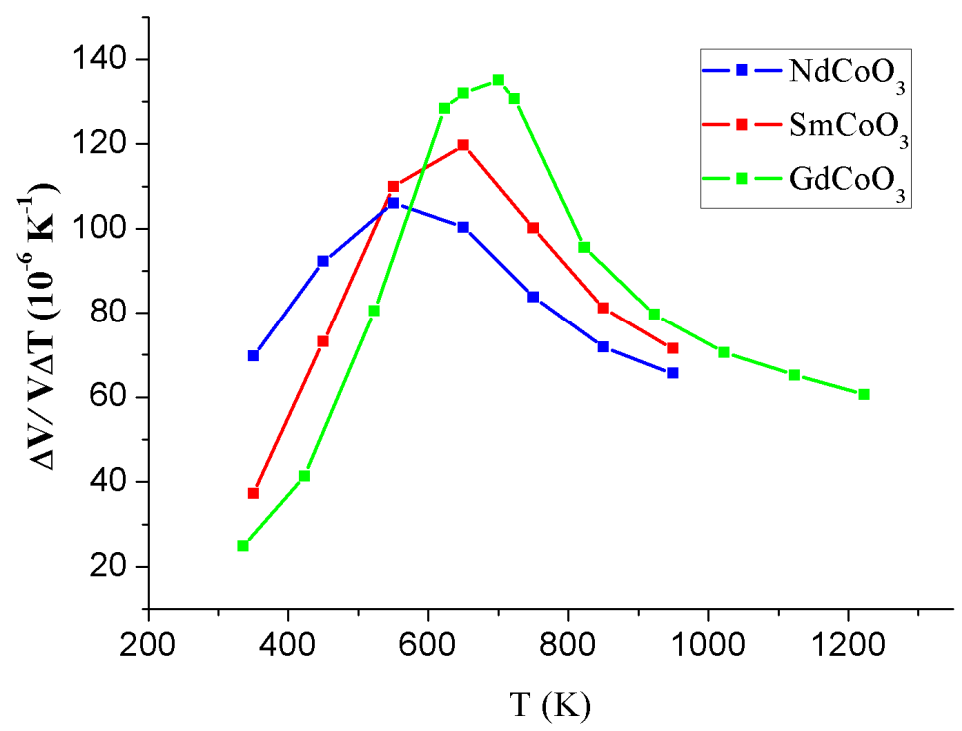

Figure 2. Temperature dependences of volume expansion coefficients for $\mathrm{NdCoO}_{3}, \mathrm{SmCoO}_{3}$, and $\mathrm{GdCoO}_{3}$. The data for $\mathrm{GdCoO}_{3}$ are taken from [39].

The given dependences are characterized by the presence of maxima within $550 \mathrm{~K}$ for $\mathrm{NdCoO}_{3}$ and $650 \mathrm{~K}$ for $\mathrm{SmCoO}_{3}$.

The combined analysis of the specific heat and thermal expansion of rare earth cobalt oxides and their solid solutions demonstrated that their temperature dependence exhibits characteristic anomalies related to the occupation of the high-spin states of cobalt ions and to the additional electron contribution arising at the insulator-metal transition occurring with the growth of the temperature. With the decrease in the radius of the rare earth ion or with the growth of chemical pressure, the spin gap in these compounds grows and is sample dependent, so we observed the shift of the low-temperature feature toward higher temperatures and the gradual merging of the two contributions to the specific heat and thermal expansion [43]. In Figure 2 the high-temperature feature of the thermal expansion of $\mathrm{NdCoO}_{3}, \mathrm{SmCoO}_{3}$, and $\mathrm{GdCoO}_{3}$ due to the insulator-metal transition observed for the entire series of rare-earth cobalt oxides with a characteristic transition temperature $T_{I M}$ increasing with decreasing ion radius of the rare-earth element is shown. $T_{I M}$ increasing determine the origin of the sample dependence in the volume expansion (Figure 2).

\subsection{Magnetic Properties}

The temperature dependences of the molar magnetic susceptibility $\chi(T)$ and field dependences of the magnetic moment $\mathrm{M}(\mathrm{H})$ of the $\mathrm{NdCoO}_{3}$ and $\mathrm{SmCoO}_{3}$ samples are shown in Figure 3. The magnetic moment values of $\mathrm{NdCoO}_{3}$ are almost fifteen times higher than the similar values for $\mathrm{SmCoO}_{3}$. The magnetization curves obtained in the FC and ZFC modes do not differ from each other for both samples. In contrast to the behavior of the $\mathrm{NdCoO}_{3}$ magnetic susceptibility, being reduced progressively with increasing temperature in the entire studied range (Figure 3a), the $\mathrm{SmCoO}_{3}$ susceptibility is characterized by a plateau in the temperature range $180-270 \mathrm{~K}$. A further temperature raise leads to an increase in the $\mathrm{SmCoO}_{3}$ magnetic susceptibility (Figure 3b) which is caused by the appearance of a contribution from $\mathrm{Co}^{3+}$ ions at high temperatures. The temperature dependence of the magnetic susceptibility of $\mathrm{RCoO}_{3}$ is determined by the magnetization of rare-earth ions and the additional paramagnetic contribution induced by the thermally excited magnetic terms of $\mathrm{Co}^{3+}$ ions. The obtained experimental data are in good agreement with similar studies in recently published works $[44,45]$. 


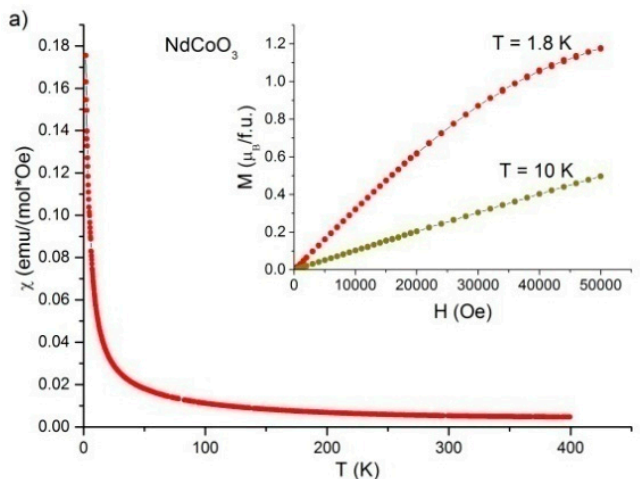

(a)

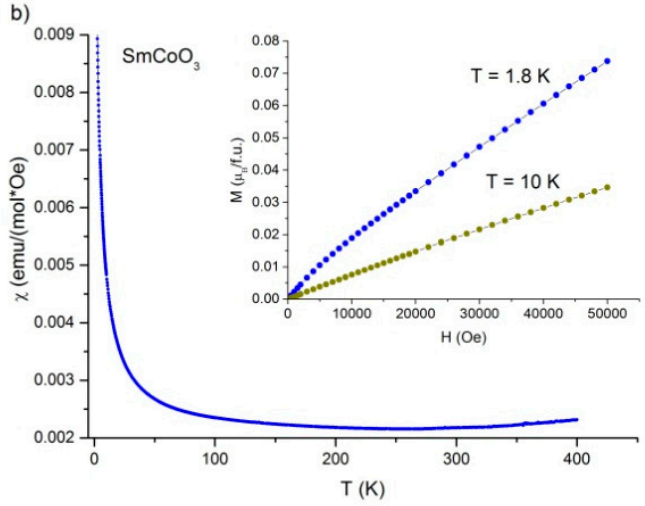

(b)

Figure 3. Temperature dependences of the molar magnetic susceptibility for $\mathrm{NdCoO}_{3}(\mathbf{a})$ and $\mathrm{SmCoO}_{3}$ (b) samples $(H=15000 \mathrm{Oe})$. The insets show the magnetization curves at $T=1.8$ and $10 \mathrm{~K}$.

The field dependences of magnetization correspond to paramagnetic behavior (Figure 3, insets), while maintaining the linearity in the region of weak fields. The magnetization saturation trends at $T=1.8 \mathrm{~K}$ are not observed in the entire field range up to $50000 \mathrm{Oe}$ for both samples.

The temperature dependences of the inverse molar susceptibility $1 / \chi$ of the $\mathrm{NdCoO}_{3}$ samples (Figure 4a, inset) and $\mathrm{SmCoO}_{3}$ (Figure $4 \mathrm{~b}$, inset) are shown in Figure 4 . In the entire temperature range under consideration, these dependences do not obey the Curie-Weiss law. Taking into account the linearity of temperature dependence of the reduced magnetic susceptibility $\chi T$ in a certain temperature range (Figure 4), it is worth describing the magnetic sample properties in order to divide the temperature range into a low-temperature, high-temperature, and intermediate interval where dependence is linear.

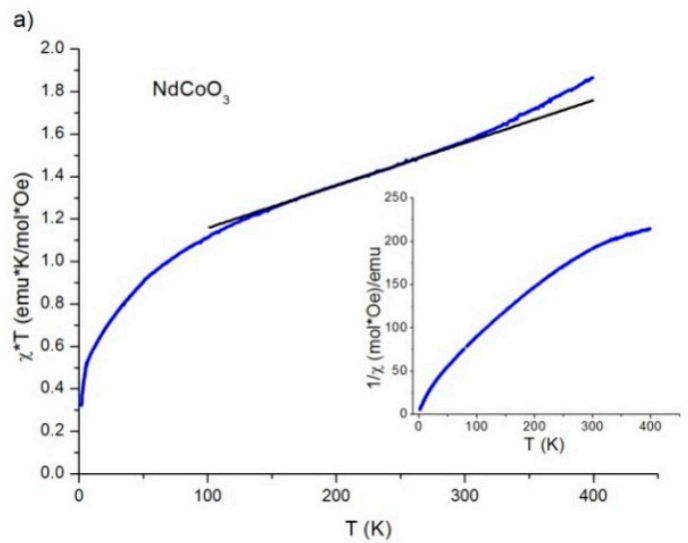

(a)

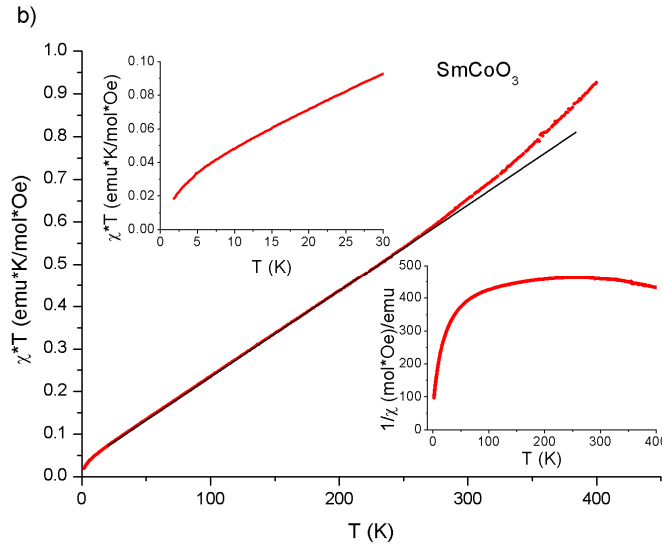

(b)

Figure 4. Temperature dependences of the reduced magnetic susceptibility $\chi \mathrm{T}$ for $\mathrm{NdCoO}_{3}(\mathbf{a})$ and $\mathrm{SmCoO}_{3}(\mathbf{b})$ samples. The temperature dependences of the inverse magnetic susceptibility $\left(\mathrm{NdCoO}_{3}\right.$ (a), $\left.\mathrm{SmCoO}_{3}(\mathbf{b})\right)$ and the $\chi \mathrm{T}$ dependence for $\mathrm{SmCoO}_{3}$ in the low-temperature region (b) are presented in the insets.

The $\chi T$ dependences in the intermediate temperature range were approximated by straight lines with convergence coefficients $R$ equal to 0.99999 for $\mathrm{SmCoO}_{3}$ and 0.99913 for $\mathrm{NdCoO}_{3}$. The linearity of the $\chi T$ dependence allows one to describe the magnetic susceptibility in the interval as a superposition of two contributions: $\chi=C / T+\chi_{V V}$, where $C / T$ is the orientation paramagnetic Curie susceptibility 
of rare-earth ions, and $\chi_{V V}$ is the Van Vleck polarization susceptibility. The diamagnetic contribution of the electron shells is sufficiently small, and therefore is ignored.

The temperature ranges, the calculated values $C, \chi_{V V}$ obtained by experiment and effective magnetic moments $\mu_{\text {eff }}^{\text {exp }}$ for $\mathrm{NdCoO}_{3}$ and $\mathrm{SmCoO}_{3}$ compounds in the intermediate temperature range $T_{\min }-T_{\max }$ are shown in Table 2. Some theoretical values are also given [46].

Table 2. and $T_{\max }$ are boundaries of the temperature range, where the $\chi T$ dependence is linear, $C$ is the Curie constant of orientation paramagnetic susceptibility, $\chi_{V V}$ is the Van Vleck polarization susceptibility, $\mu_{e f f}^{\exp }$ is the value of the experimentally obtained effective magnetic moment, $\mu_{e f f}^{\text {teor }}$ is the theoretical value of the effective magnetic moment, $\mu_{e f f}^{\text {teor }(\mathrm{VV})}$ is the theoretical value of the effective magnetic moment taking into account the Van Vleck paramagnetism, $R$ is the convergence coefficient of the experimental data and the fitting line in the given temperature range.

\begin{tabular}{lcccccccc}
\hline & $T_{\min }(\mathrm{K})$ & $T_{\max }(\mathrm{K})$ & $C\left(\frac{e m u \cdot K}{m o l \cdot O e}\right)$ & $\chi_{V V}\left(\frac{e m u}{m o l \cdot O e}\right)$ & $\mu_{e f f}^{\text {exp }} \mu_{\mathrm{B}}$ & $\mu_{\text {eff }}^{\text {teor }} \mu_{\mathrm{B}}$ & $\mu_{e f f}^{\text {teor(VV) }} \mu_{\mathrm{B}}$ & $R$ \\
\hline $\mathrm{NdCoO}_{3}$ & 165 & 250 & 0.956 & 0.00201 & 2.77 & 3.62 & 3.68 & 0.99993 \\
\hline $\mathrm{SmCoO}_{3}$ & 15 & 270 & 0.03504 & 0.00202 & 0.53 & 0.84 & 1.55 & 0.99999 \\
\hline
\end{tabular}

The obtained values of $C$ and $\mu_{e f f}^{\exp }$ are a little larger than the similar values obtained in [47] for $\mathrm{SmCoO}_{3}\left(C=0.0276 \mathrm{emu} \cdot \mathrm{K} /(\mathrm{mol} \cdot \mathrm{Oe}), \mu_{\text {eff }}=0.47 \mu_{\mathrm{B}}\right)$. The effective magnetic moments for both $\mathrm{Nd}^{3+}$ and $\mathrm{Sm}^{3+}$ are significantly lower than their theoretical values calculated for free ions (Table 2).

The total magnetic susceptibility of $\mathrm{NdCoO}_{3}$ and $\mathrm{SmCoO}_{3}$ can be represented as a sum of two independent summands (since the Co ions acquire a magnetic moment only at high temperatures, the exchange interaction of Co-RE can be neglected)

$$
\chi_{\mathrm{Sm}(\mathrm{Nd}) \mathrm{CoO}_{3}}=\chi_{\mathrm{Sm}(\mathrm{Nd})}+\chi_{\mathrm{Co}}
$$

where $\chi_{S m(N d)}$ and $\chi_{C o}$ are the magnetic susceptibilities of samarium (neodymium) and cobalt ions, respectively. To describe the contribution of cobalt ions to the total magnetization of $\mathrm{Sm}(\mathrm{Nd}) \mathrm{CoO}_{3}$, a diagram of the $\mathrm{Co}^{3+}$ ion levels in a crystal field taking into account the spin-orbit interaction is shown in Figure 5. The ground term is a ${ }^{1} A_{1}$ low-spin singlet, separated from the triplet sublevel $\widetilde{J}=1$ of the ${ }^{5} T_{2}$ high-spin state by the $\Delta_{S}$ spin gap. At $\Delta_{S}=150 \mathrm{~K}$, the position of the terms for $\mathrm{LaCoO}_{3}$ corresponds to $[13,23,48]$. Lanthanum substitution on another rare-earth ion with a smaller ionic radius leads to a chemical pressure generation that is equivalent to the external pressure. Therefore, the substitution will lead to the additional stabilization of low-spin state or, in other words, to increase the spin gap.

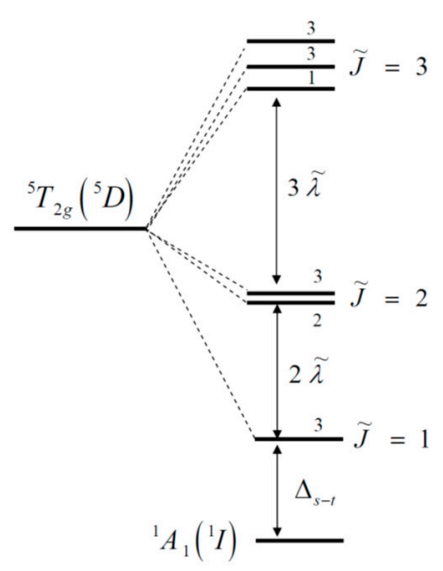

Figure 5. A set of low-energy terms for the $\mathrm{d}^{6}$ electronic configuration for $\mathrm{Co}^{3+}$ ion in a crystalline field of octahedral symmetry, taking into account spin-orbit interaction. The degeneracy multiplicity is shown by digits for terms [15]. 
At low temperatures, there are the $\mathrm{SmCoO}_{3}$ and $\mathrm{NdCoO}_{3}$ cobalt ions in the ${ }^{1} A_{1}$ nonmagnetic low-spin state. With increasing temperature, thermal excitations of the high-spin state with a nonzero magnetic moment (the multiplicity fluctuations) and increase of the magnetization occur. The statistical sum of the $\mathrm{Co}^{3+}$ ions per mole of substance can be represented as:

$$
Z=\left[1+e^{-\beta \Delta_{S}}+2 e^{-\beta \Delta_{S}} \operatorname{ch}\left(g_{1} \mu_{B} \widetilde{B} \beta\right)+e^{-\beta\left(\Delta_{S}+2 \widetilde{\lambda}_{C_{0}}\right)}+2 e^{-\beta\left(\Delta_{S}+2 \widetilde{\lambda}_{C o}\right)} \operatorname{ch}\left(g^{\prime}{ }_{2} \mu_{B} \widetilde{B} \beta\right)+2 e^{-\beta\left(\Delta_{S}+2 \widetilde{\lambda}_{C O}\right)} \operatorname{ch}\left(g^{\prime \prime}{ }_{2} \mu_{B} \widetilde{B} \beta\right)\right]^{N_{A}}
$$

where $\widetilde{\lambda}_{C o}=185 \mathrm{~K}[48]$ is the effective constant of spin-orbit interaction, $N_{A}$ is the Avogadro number, $\widetilde{B}$ is the applied external magnetic field, $k_{B}$ is the Boltzmann constant, $\beta=1 / k_{B} T, \mu_{B}$ is the Bohr magneton, the Landé factors $g_{1}=3.4$ for the triplet $\widetilde{J}=1$ and $g_{2}^{\prime}{ }_{2}=3.1, g^{\prime \prime}{ }_{2}=1.8$ for the quintet $\widetilde{J}=1$. With the partition function, the free energy $F=-k_{B} T \ln Z$ and magnetization $M=-\partial F / \partial \widetilde{B}$ are found in a standard way. For not too low temperatures and not too strong magnetic fields, the expression for the molar magnetic susceptibility of $\mathrm{Co}^{3+}$ ions $\chi_{\mathrm{C}_{0}}=\partial M / \partial \widetilde{B}$ is as follows:

$$
\chi_{C o}=N_{A} 2 \mu_{B}^{2} \beta\left[g_{1}^{2} e^{-\beta \Delta_{S}}+g^{\prime 2} e^{-\beta\left(\Delta_{S}+2 \widetilde{\lambda}\right)}+g^{\prime \prime 2} e^{-\beta\left(\Delta_{S}+2 \widetilde{\lambda}\right)}\right] /\left[1+3 e^{-\beta \Delta_{S}}+5 e^{-\beta\left(\Delta_{S}+2 \widetilde{\lambda}\right)}\right]
$$

In the case when $\Delta_{S}>1000 \mathrm{~K}$, the spin-orbit interaction can be neglected, the expression (3) takes the form:

$$
\chi_{C o}=N_{A} \frac{g^{2} \mu_{B}^{2} S(S+1)}{3 k_{B} T} n_{H S}
$$

where $g=2$ is the Lande spin factor, $n_{H S}=\frac{g_{H S} \exp \left(-\Delta_{S} / k_{B} T\right)}{1+g_{H S} \exp \left(-\Delta_{S} / k_{B} T\right)}$ is the population of the HS- state, $g_{H S}=(2 S+1)(2 L+1)=15$ for the high-spin state with $S=2, L=1$.

The calculation results $\chi_{\mathrm{Co}}$ for $\mathrm{NdCoO}_{3}$ and $\mathrm{SmCoO}_{3}$ are given in Figure 6. The following values are used: $g_{H S}=15, \Delta_{S}=2300 \mathrm{~K}\left(\mathrm{SmCoO}_{3}\right)$ and $\Delta_{S}=1600 \mathrm{~K}\left(\mathrm{NdCoO}_{3}\right)$.

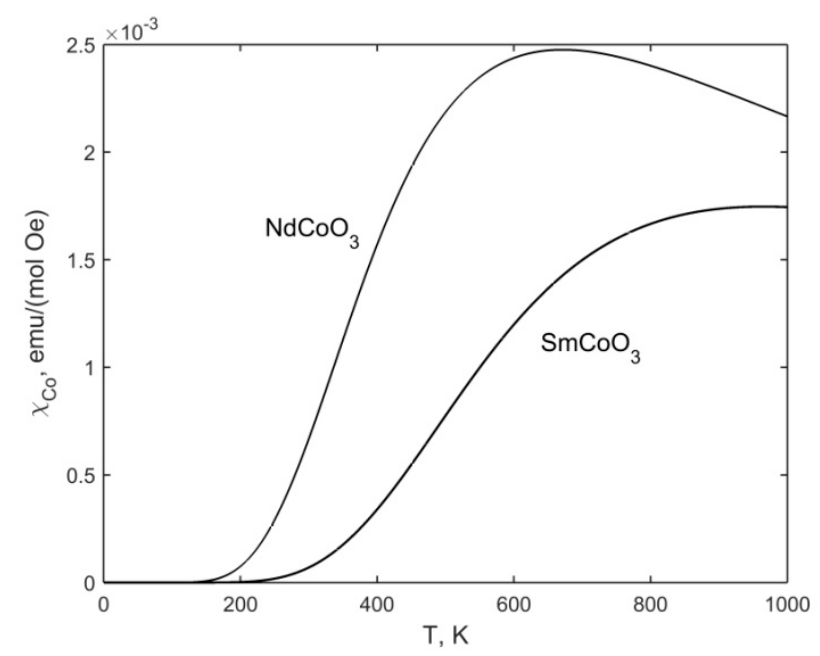

Figure 6. Temperature dependences of the $\mathrm{Co}^{3+}$ ion magnetic susceptibility for $\mathrm{NdCoO}_{3}$ and $\mathrm{SmCoO}_{3}$ samples.

The magnetic susceptibility of $\mathrm{Sm}^{3+}$ ions in $\mathrm{SmCoO}_{3}$ can be represented by the formula [49].

$$
\chi_{S m}=\frac{0.2482}{x T} \frac{1.07 x+3.67+(21.45 x+0.82) e^{-7 x / 2}+\ldots}{3+4 e^{-7 x / 2}+\ldots}
$$

where $x=\lambda_{S m} / T, \lambda_{S m}$ is the spin-orbit coupling constant of the rare-earth $\mathrm{Sm}^{3+}$ ion. It is known from spectroscopic data that the nearest excited term ${ }^{6} \mathrm{H}_{7 / 2}$ of the $\mathrm{Sm}^{3+}$ ion is separated from the main one by the energy interval $\Delta=7 / 2 \lambda_{S m}$ approximately $1000 \mathrm{~cm}^{-1}$ [49], therefore $\lambda_{S m} \approx 400 \mathrm{~K}$. Despite the fact 
that formula (5) was obtained for a free rare-earth ion with no crystalline field, however, it is possible to describe the temperature dependence of the $\mathrm{SmCoO}_{3}$ magnetic susceptibility as will be seen below.

Within low temperatures, the expression (5) takes a simpler form:

$$
\chi_{S m} \approx C / T+\chi_{V V}
$$

where $C$ is the effective Curie constant, and $\chi_{V V}$ is the Van Vleck susceptibility.

The calculation results $\chi \mathrm{T}$ and $\chi^{-1}$ respectively, for $\mathrm{SmCoO}_{3}$ (red solid line), using (1), taking into account (4) and (5) are presented in Figure 7a,b. In contrast, in Figure 7, the contribution of only $\mathrm{Sm}^{3+}$ ions, according to (5) is depicted by the blue dashed line. That can be seen to be in a good agreement with the experiment. The theoretical dependence $\chi T$ (Figure 7a) shows a smooth deviation from the linear dependence at $T \approx 125 \mathrm{~K}$ (shown by the dashed arrow), below this temperature the expression (6) is valid. Nevertheless, the approximation (6) for $\mathrm{SmCoO}_{3}$ can be seen from Figure $4 \mathrm{~b}$ to be valid over a wide temperature range up to $250 \mathrm{~K}$ (see above). Thus, $\mathrm{Co}^{3+}$ magnetic moment is provided above 250K for $\mathrm{SmCoO}_{3}$ that agrees with Figure 6.

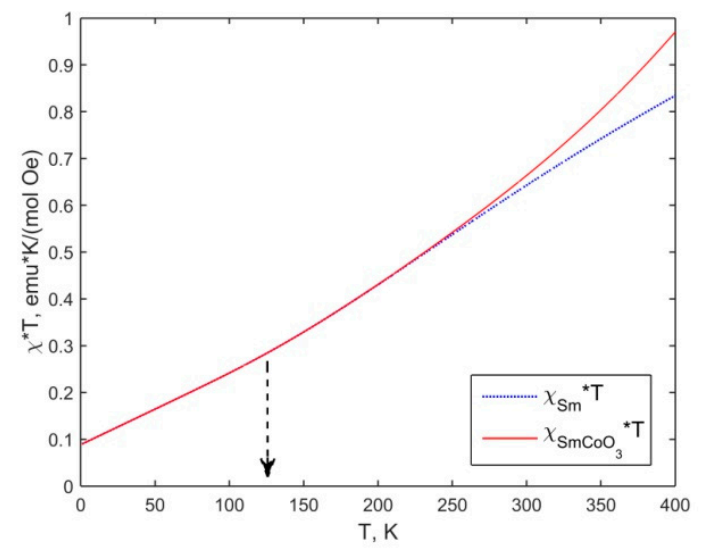

(a)

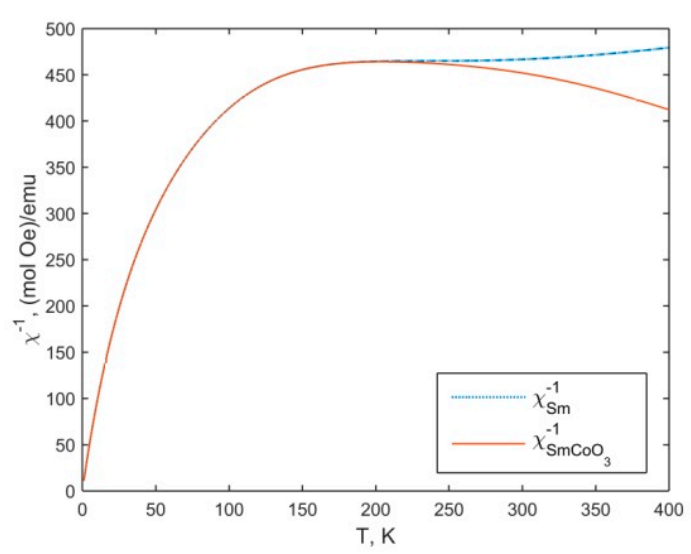

(b)

Figure 7. The calculation results of the temperature dependences $\chi T$ (a) and $\chi^{-1}(\mathbf{b})$, respectively, for $\mathrm{SmCoO}_{3}$ (red solid line). By contrast, the contribution of only $\mathrm{Sm}^{3+}$ ions is shown by blue dashed line.

The ground state of free $\mathrm{Nd}^{3+}$ ion with the $4 f^{3}$ electronic configuration is the ${ }^{4} \mathrm{I}_{9 / 2}$ multiplet $(\mathrm{L}=6$, $\mathrm{S}=3 / 2$ ). The nearest excited state ${ }^{4} \mathrm{I}_{11 / 2}$ is $1900 \mathrm{~cm}^{-1}$ higher in energy. As for the free $\mathrm{Sm}^{3+}$ ion, its electronic configuration is $4 f^{5}$, the ground multiplet state is ${ }^{6} \mathrm{H}_{5 / 2}(\mathrm{~L}=5, \mathrm{~S}=5 / 2)$. The distinctive feature of this ion is the relative proximity to the first excited state ${ }^{6} \mathrm{H}_{7 / 2}$. The energy difference of these states for free $\mathrm{Sm}^{3+}$ ion is approximately $1000 \mathrm{~cm}^{-1}$ [49].

The magnetic properties for ions essentially depend on their environment, i.e., on the crystal field value and symmetry. Hence, in perovskite-like crystals, such as cobaltites, the rare-earth ion is in a low-symmetrical ligand environment. The field of such symmetry splits the main $\mathrm{Sm}^{3+}$ multiplet into three and five Kramers doublets for $\mathrm{Nd}^{3+}$, with each of them having a certain magnetic moment. In the general case, such a splitting leads to a decrease or, by contrast, an increase in the magnetic ion moment and, in addition, the influence of the crystal field can be expressed in a significant difference between the $g$-factor of Kramers doublets and $g_{0}=\frac{3}{2}-\frac{L(L+1)-S(S+1)}{2 J(J+1)}$ for the free ion and their strong anisotropy, which turns to result in magnetic susceptibility anisotropy. For polycrystalline samples, the average magnetic susceptibility can be calculated as $\langle\chi\rangle=\left(\chi_{\|}+2 \chi_{\perp}\right) / 3$, where $\chi_{\|}$and $\chi_{\perp}$ are the susceptibility components in parallel and perpendicular directions to the external applied magnetic field. Therefore, the complex energy level structure of the $\mathrm{Sm}^{3+}$ and $\mathrm{Nd}^{3+}$ ions in a crystal field of low 
symmetry leads to the temperature dependence in the low-temperature region $\left(T<T_{\min }\right)$ shown in Figure 4.

It has been known that in order to calculate the temperature dependence of magnetization and susceptibility, the positions of the energy levels of the $E_{n}$ system taking into account the external magnetic field have to be realized. Van Fleck (1932) studied the energy contributions in terms ofperturbative approach depending on the effect of the magnetic field $H: E_{n}=E_{n}^{(0)}+H E_{n}^{(1)}+H^{2} E_{n}^{(2)}$, where $E_{n}^{(0)}$ are the energy system levels without the external magnetic field $\hat{H}_{0}|n\rangle=E_{n}^{(0)}|n\rangle$, generally developing the groups of degenerate states; $E_{n}^{(1)}=\left\langle n\left|\mu_{B}\left(\hat{L}_{s}+g_{0} \hat{S}_{s}\right)\right| n\right\rangle, E_{n}^{(2)}=\sum_{n^{\prime} \neq n} \frac{\left|\left\langle n\left|\mu_{B}\left(\hat{L}_{s}+g_{0} \hat{S}_{s}\right)\right| n^{\prime}\right\rangle\right|^{2}}{E_{n}-E_{n}^{\prime}}$ are Zeeman coefficients of the first and second order (the $z$ axis is directed along the magnetic field). The Hamiltonian $\hat{H}_{0}$ contains inter-electron repulsion, spin-orbit interaction, and crystal field energy.

The temperature dependence equation of magnetic susceptibility, known as the Van Vleck equation [50], has the form: $\chi_{\text {Vleck }}=N_{A} \frac{\sum_{n}\left[\frac{\left(E_{n}^{(1)}\right)^{2}}{k_{B} T}-2 E_{n}^{(2)}\right] \exp \left(-\frac{E_{n}^{(0)}}{k_{B} T}\right)}{\sum_{n} \exp \left(-\frac{E_{n}^{(0)}}{k_{B} T}\right)}$, where $N_{A}$ is the Avogadro constant, and $k_{B}$ is the Boltzmann constant. It is generally accepted that, in commonly used $H \leq 10 \mathrm{kOe}$ fields, the Zeeman interaction energy is usually less than the splitting caused by inter-electron repulsion, crystal field, and spin-orbit interaction; however, in case of rare-earth ions in low-symmetry crystal fields, the multiplet splitting into Kramers sublevels being sufficiently close to each other (see above) seems to be compared with the interaction energy with magnetic field and to be observed by an unusual dependence of paramagnetic Van Vleck susceptibility on the magnetic field value. Thus, to characterize properly the magnetic properties of the rare-earth and transition metal ions in low-symmetry fields, both the low-symmetry part of crystal field and the magnetic field influence have to be similarly considered or, in other words, simultaneously taken into account.

In contrast to $\mathrm{SmCoO}_{3}, \mathrm{NdCoO}_{3}$ has a linear region $\chi \mathrm{T}$ and $\chi^{-1}$ in a more narrow temperature range (see Table 2 ). Above $T_{\max } \approx 250 \mathrm{~K}$, the contribution of $\mathrm{Co}^{3+}$ ions to the total magnetic susceptibility of the sample becomes detectible for $\mathrm{NdCoO}_{3}$.

In a low-symmetry crystal field, the main term ${ }^{6} \mathrm{H}_{5 / 2}$ of the $\mathrm{Sm}^{3+}$ ion splits into three Kramers doublets, and the main term ${ }^{4} \mathrm{I}_{9 / 2}$ of the $\mathrm{Nd}^{3+}$ ion splits into five ones in a wider energy range [49]. This causes a significant difference in the $T_{\min }$ temperature for $\mathrm{SmCoO}_{3}$ and $\mathrm{NdCoO}_{3}$ (see Table 2). As otherwise stated, with decreasing temperature for $\mathrm{NdCoO}_{3}$, crystal field effects are important even at $T_{\min } \approx 165 \mathrm{~K}$, while for $\mathrm{SmCoO}_{3}$ only at $T<T_{\min } \approx 15 \mathrm{~K}$.

The origin of Van Vleck paramagnetism is to add the wave functions of thermally unpopulated excited states to the wave functions of ground state. The Van Vleck susceptibility of the free $\mathrm{Sm}^{3+}$ $\left(\mathrm{Nd}^{3+}\right)$ ions is due to possible (virtual) quantum transitions between the energetically lowest ${ }^{6} \mathrm{H}_{5 / 2}$ $\left({ }^{4} \mathrm{I}_{9 / 2}\right)$ state and the nearest excited ${ }^{6} \mathrm{H}_{7 / 2}\left({ }^{4} \mathrm{I}_{11 / 2}\right)$ state. In the crystal field, additional multiplet splitting occurs and, besides the indicated transitions, some possible ones within the same multiplet have to be taken into account. This is the reason for the difference between the Van Vleck susceptibility of $\mathrm{SmCoO}_{3}$ and $\mathrm{NdCoO}_{3}$ and that for free ions.

The electronic structure of cobaltites calculated in the framework of the LDA + GTB multi-electron approach $[17,39]$ depends on the $n_{H S}$ concentration. Therefore, correlation of the changes in activation energy with changes in thermal expansion and magnetic susceptibility is also due to the contribution of an increasing concentration of high-spin states with temperature rise.

\subsection{Thermal Expansion}

The experimental temperature dependences of the volume thermal expansion coefficient $\beta(T)$, obtained in the heating and cooling modes are presented in Figure 8. Hysteretic phenomena were not observed. The coefficient $\beta$ for $\mathrm{NdCoO}_{3}$ compound is characterized by the presence of two diffuse anomalies near 400 and $600 \mathrm{~K}$, and for $\mathrm{SmCoO}_{3}$ by one maximum near $650 \mathrm{~K}$ (Figure $8 \mathrm{a}$ ). 
Deviation from the usual linear contribution due to anharmonicity occurs in the temperature ranges of 250-270 K and 310-330 K for $\mathrm{NdCoO}_{3}$ and $\mathrm{SmCoO}_{3}$, observed in the temperature dependences of the deformation $\Delta L / L$ (Figure $8 \mathrm{~b}$ ). In this case, the temperatures of the first maximum for $\mathrm{NdCoO}_{3}$ and the maximum for $\mathrm{SmCoO}_{3}$ correlate with the maxima obtained on the temperature dependences of the thermal expansion during diffraction studies. The anomalous contribution of electronic origin due to multiplicity fluctuations is revealed by the deviations from linear behavior shown in the inset to Figure 8 .

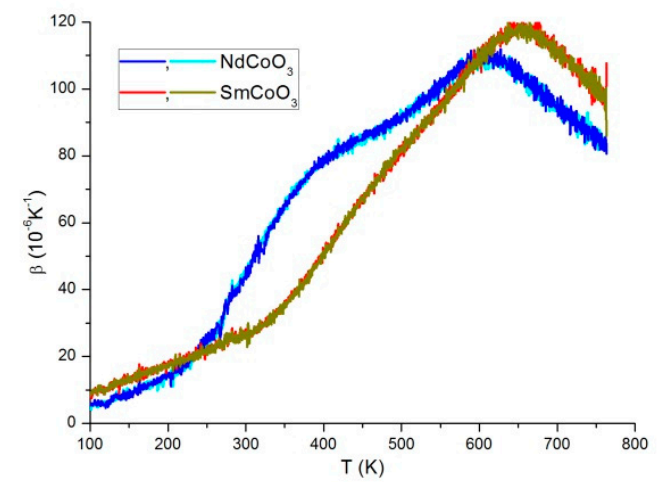

(a)

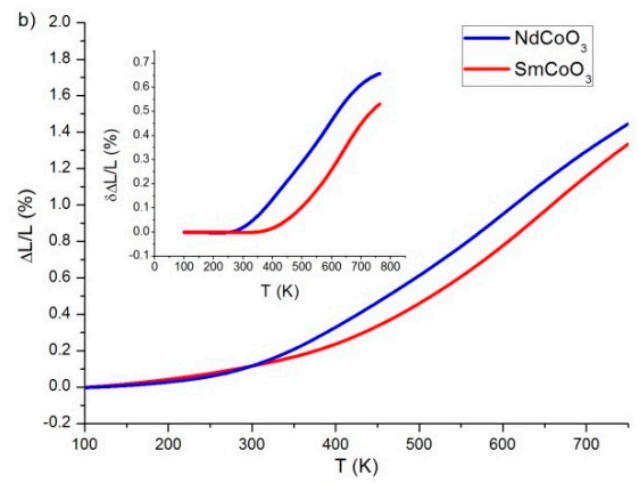

(b)

Figure 8. Temperature dependences of the volume thermal expansion coefficient $\beta$, obtained as a result of successive heating-cooling cycles $(\mathbf{a})$ and $(\Delta L / L)$ deformation (b), for $\mathrm{NdCoO}_{3}$ and $\mathrm{SmCoO}_{3}$ samples. The inset shows abnormal contributions to the deformation after subtraction of the standard linear contribution to the lattice expansion.

In the case of spin crossover materials, a large contribution to the anomaly of thermal expansion is made by the redistribution of the HS/LS statistical weights due to the large difference in their ionic radii [39]; therefore, the unit cell volume as a temperature function can be represented as

$$
V(T)=V_{H S}(T) n_{H S}(T)+V_{L S}(T) n_{L S}(T)
$$

where, $V_{H S}(T), V_{L S}(T)$ is the unit cell volume, respectively, in the phase of the HS- and LS- states, $n_{H S / L S}(T)$ is the population of HS/LS- states., this turns to be represented as

$$
V_{H S}(T)=V_{H S}^{(0)}\left(1+\beta_{H S} T\right)
$$

and

$$
V_{L S}(T)=V_{L S}^{(0)}\left(1+\beta_{L S} T\right)
$$

where $\beta_{H S / L S}$ is the volumetric thermal expansion coefficient, and $V_{H S / L S}^{(0)}$ is the unit cell volume when $T=0$, respectively, in the phase of the HS/LS- state. In the case of rare-earth cobalt oxides, the non-magnetic LS- state is the ground state of cobalt ion, and the HS-state is possible with increasing temperature, therefore expression (8) is suggested to be written by the so-called «virtual crystal model», when the ground state of cobalt ions is the artificially created HS-state (hypothetical HS-phase). A similar approach was previously used to describe the thermodynamic and magnetic properties of $\mathrm{GdCoO}_{3}$ [39], where $V_{H S / L S}^{(0)}$ was determined by first-principle calculations using the DFT method. Since $n_{L S}(T)=1-n_{H S}(T)$, then

$$
V(T)=\left(V_{H S}^{(0)}-V_{L S}^{(0)}\right) n_{H S}(T)+\left(V_{H S}^{(0)} \beta_{H S}-V_{L S}^{(0)} \beta_{L S}\right) T n_{H S}(T)+V_{L S}^{(0)}\left(1+\beta_{L S} T\right)
$$


In expression (10), both the background (regular) contribution, i.e., the second and third summands due to the anharmonicity of lattice vibrations in the phase of mixed LS/HS- and pure LS- states, and the anomalous contribution of thermal expansion, i.e., the first summand arising due to multiplicity fluctuations of cobalt ions can be distinguished. Since the characteristic values are $\beta_{H S / L S} \sim 10^{-5}$ $1 / \mathrm{K}$, and $0 \leq n_{H S / L S}(T)<1$ then at $T<1000 \mathrm{~K}$, the first summand makes the largest contribution compared to the second one, therefore, the volumetric thermal expansion coefficient can be represented as $\beta=\frac{1}{V} \frac{\partial V}{\partial T} \approx \delta \beta+\beta_{\text {reg }}$, where $\delta \beta=\frac{\left(V_{H S}^{(0)}-V_{L S}^{(0)}\right)}{V_{L S}^{(0)}} \frac{\partial n_{H S}(T)}{\partial T}$. Thus, the anomalous contribution to the volumetric thermal expansion coefficient is proportional to the first-order derivative with respect to the population temperature of the HS- state $n_{H S}$ and is determined by the magnitude of spin gap. In Figure 9, by comparison, the experimental data of anomalous contribution to thermal expansion and the calculated values $\partial n_{H S} / \partial T$ for $\mathrm{NdCoO}_{3}$ and $\mathrm{SmCoO}_{3}$ at $\Delta_{S}=1600$ and $2300 \mathrm{~K}$, respectively, are presented.

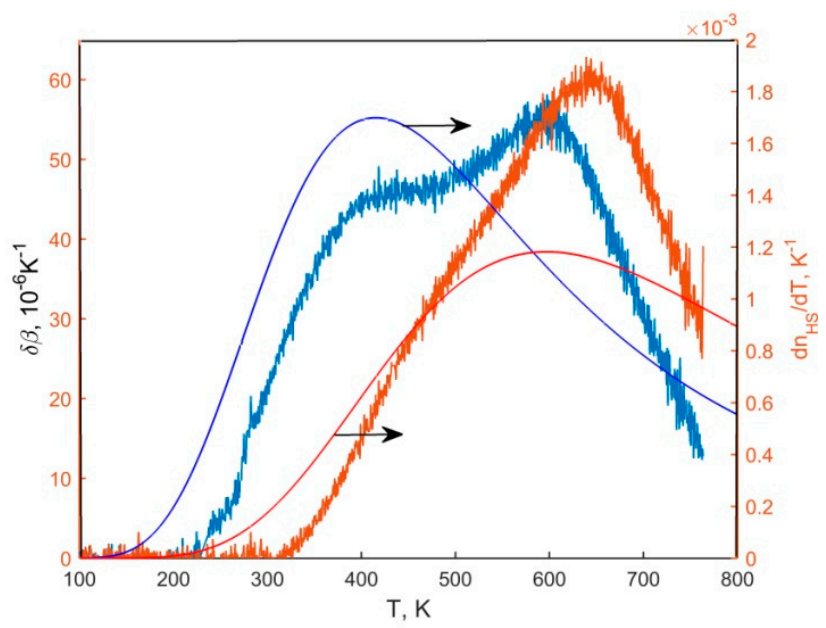

Figure 9. Anomalous contributions to the volumetric thermal expansion coefficient for $\mathrm{NdCoO}_{3}$ (depicted by blue color) and $\mathrm{SmCoO}_{3}$ (depicted by red color). Solid blue and red lines show the calculated dependences $\partial n_{\mathrm{HS}} / \partial \mathrm{T}$ for $\mathrm{NdCoO}_{3}$ and $\mathrm{SmCoO}_{3}$, respectively.

Figures $8 \mathrm{a}$ and 9 illustrate that, in contrast to $\mathrm{SmCoO}_{3}$, in temperature dependence of the volumetric thermal expansion coefficient of $\mathrm{NdCoO}_{3}$, two maxima can be clearly distinguished. The first (low-temperature) maximum is associated with fluctuations of the spin multiplicity of cobalt ions, and the second one with the insulator-semimetal transition (crossover) observed for all rare-earth cobalt oxides with increasing temperature and characteristic transition temperature of the rare-earth element. For $\mathrm{SmCoO}_{3}$, the spin gap obtained above by the magnetic data analysis is large enough, thus both peaks almost coincide. A similar situation is observed for solid solutions of rare-earth cobaltites. Then, in $\mathrm{La}_{1-x} \mathrm{Gd}_{x} \mathrm{CoO}_{3}$, the low-temperature maximum of thermal expansion shifts to the region of higher temperatures with an increase in the gadolinium concentration and gradually coincides with the second maximum [43].

\subsection{Transport Properties}

The temperature dependences of the electrical resistivity $\rho(T)$ for $\mathrm{NdCoO}_{3}$ and $\mathrm{SmCoO}_{3}$ samples and the dependence of resistivity logarithm on the reciprocal temperature are shown in Figure 10. The $\rho(T)$ dependences reliably correspond to the semiconductor type $d \rho(T) / d T<0$ over the studied 300 to $750 \mathrm{~K}$ range. According to the $\ln \rho(1 / T)$ dependences, it is matter of fact that the semiconductor type of conductivity can be described using the currently accepted thermal activation relation of the form $\rho(T)=\rho_{\infty} \exp \left(E_{a} / k_{B} T\right)$, where $E_{a}$ is the activation energy [51], and $\rho_{\infty}$ is the constant determined by $T \rightarrow \infty$. Moreover, for each sample, there is a temperature $T^{*}$ when the activation energy changes 
in the intermediate region and temperature $T^{* *}$ characterizing the variation from the activation law at high temperatures. The values of the obtained parameters are presented in Table 3.

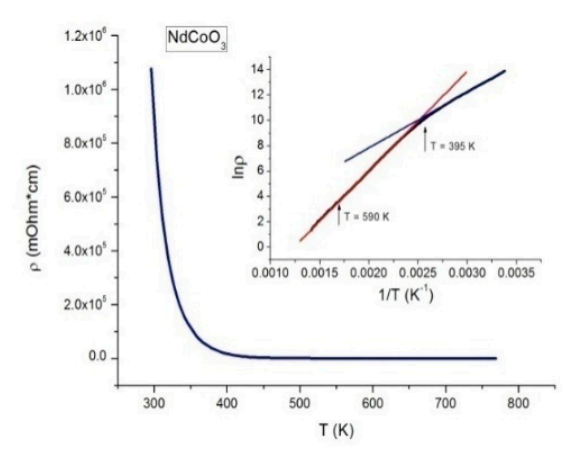

(a)

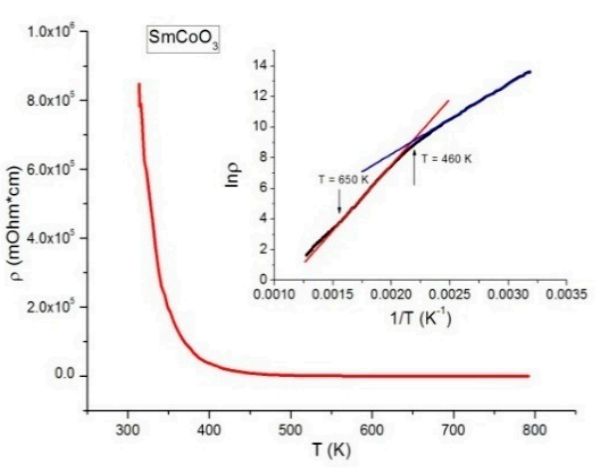

(b)

Figure 10. Temperature dependence of electrical resistivity for the $\mathrm{NdCoO}_{3}$ (a) and $\mathrm{SmCoO}_{3}$ (b) samples. The corresponding dependencies of the resistivity logarithm on the reciprocal temperature are illustrated in the insets. Straight lines show accordance with the thermo-activation law (blue-the region of intermediate temperatures, red-high temperatures).

Table 3. The parameters describing the thermal activation conductivity of the samples and the temperatures of $\mathrm{NdCoO}_{3}$ and $\mathrm{SmCoO}_{3}$ electronic transition ( $R$ is the convergence coefficient).

\begin{tabular}{|c|c|c|c|c|c|c|c|c|}
\hline & \multirow{2}{*}{$\mathrm{T}^{*}, \mathrm{~K}$} & \multicolumn{3}{|c|}{$T<T^{*}$} & \multicolumn{3}{|c|}{$T>T^{*}$} & \multirow{2}{*}{$\mathrm{T}^{* *}, \mathrm{~K}$} \\
\hline & & $E_{a}, \mathrm{eV}$ & $\begin{array}{c}\rho_{\infty} \\
\mathrm{mOhm} \cdot \mathrm{cm}\end{array}$ & $R$ & $E_{a}, \mathrm{eV}$ & $\begin{array}{c}\rho_{\infty}, \\
\mathrm{mOhm} \cdot \mathrm{cm}\end{array}$ & $R$ & \\
\hline $\mathrm{NdCoO}_{3}$ & 395 & $0.379 \pm 0.001$ & $0.390 \pm 0.001$ & 0.99955 & $0.679 \pm 0.001$ & $5.81 \times 10^{-5}$ & 0.99991 & 590 \\
\hline $\mathrm{SmCoO}_{3}$ & 460 & $0.394 \pm 0.001$ & $0.401 \pm 0.001$ & 0.99893 & $0.739 \pm 0.001$ & $6.56 \times 10^{-5}$ & 0.99944 & 650 \\
\hline
\end{tabular}

Accordance with the thermal activation law is depicted by straight lines in the insets to Figure 10.

The temperature deviations from a thermal activation law and the characteristic temperatures of changes in the activation energy correlate with anomalies in the temperature dependences of the volumetric thermal expansion coefficient $\beta(T)$ for both samples.

\section{Conclusions}

The features of thermal expansion, magnetic susceptibility, and transport properties of $\mathrm{NdCoO}_{3}$ and $\mathrm{SmCoO}_{3}$ cobaltites being in a good agreement were experimentally demonstrated. Partially, these correlations were previously known, e.g., those of thermal expansion with a spin and electronic transition [5]. The features have been shown theoretically to be associated with a population increase of high-spin states of $\mathrm{Co}^{3+}$ ions. A comparison of the results with well- studied $\mathrm{GdCoO}_{3}$ allows one to identify both general trends inherent in all rare-earth cobaltites based on lanthanide compression and the specific properties of samples containing $\mathrm{Nd}^{3+}, \mathrm{Sm}^{3+}$ ions formed with strong single-ion anisotropy and crystal field effects at low temperatures.

A quantitative assessment of the contribution from the fluctuations of multiplicity to the magnetic properties of $\mathrm{NdCoO}_{3}$ and $\mathrm{SmCoO}_{3}$ samples seems to be a rather difficult task, since in addition to the complex structure of the energy levels of $\mathrm{Sm}^{3+}$ and $\mathrm{Nd}^{3+}$ ions in a low-symmetry crystal field, it is necessary to take into account the influence of oxygen non-stoichiometry of the samples referred to a number of research works [52,53]. On the one hand, oxygen non-stoichiometry is the main reason for defects in the structure of rare-earth cobaltites leading to the formation of magnetic excitons [54,55]; on the other hand, it can lead to dimer formation [56] and the appearance of $\mathrm{Co}^{3+}$ ions in the HS- state even at low temperatures. 
Author Contributions: The manuscript was written through contributions of all authors. All authors have read and agreed to the published version of the manuscript.

Funding: This work is supported by the Russian Science Foundation grant 18-02-00022.

Conflicts of Interest: The authors declare no conflict of interest.

\section{References}

1. Goodenough, J.B. An interpretation of the magnetic properties of the perovskite-type mixed crystals $\mathrm{La}_{1-x} \mathrm{Sr}_{x} \mathrm{CoO}_{3-\delta}$. J. Phys. Chem. Solids 1958, 6, 287-297. [CrossRef]

2. Raccah, P.M.; Goodenough, J.B. First-order localized-electron $\leftrightarrows$ Collective-electron transition in $\mathrm{LaCoO}_{3}$ Phys. Rev. 1967, 155, 932-943. [CrossRef]

3. Bhide, V.G.; Rajoria, D.S. Mössbauer studies of the high-spin-Low-spin equilibria and the localized-collective electron transition in $\mathrm{LaCoO}_{3}$. Phys. Rev. B 1972, 6, 1021-1032. [CrossRef]

4. Asai, K.; Yokokura, O.; Nishimori, N.; Chou, H.; Tranquada, J.M.; Shirane, G.; Higuchi, S.; Okajima, Y.; Kohn, K. Neutron-scattering study of the spin-state transition and magnetic correlations in $\mathrm{La}_{1-x} \mathrm{Sr}_{x} \mathrm{CoO}_{3}(x$ $=0$ and 0.08). Phys. Rev. B 1994, 50, 3025-3032. [CrossRef]

5. Knížek, K.; Jirak, Z.; Hejtmanek, J.; Veverka, M.; Marysko, M.; Maris, G.; Palstra, T.T.M. Structural anomalies associated with the electronic and spin transitions in $\mathrm{LnCoO}_{3}$. Eur. Phys. J. B Condens. Matter Complex Syst. 2005, 47, 213-220. [CrossRef]

6. Alonso, J.A.; Martinez-Lope, M.J.; de la Calle, C.; Pomjakushin, V. Preparation and structural study from neutron diffraction data of $\mathrm{RCoO}_{3}(\mathrm{R}=\mathrm{Pr}, \mathrm{Tb}, \mathrm{Dy}, \mathrm{Ho}, \mathrm{Er}, \mathrm{Tm}, \mathrm{Yb}, \mathrm{Lu})$ perovskites. J. Mater. Chem. 2006, 16, 1555-1560. [CrossRef]

7. Berggold, K.; Kriener, M.; Becker, P.; Benomar, M.; Reuther, M.; Zobel, C.; Lorenz, T. Anomalous expansion and phonon damping due to the Co spin-state transition in $\mathrm{RCoO}_{3}(\mathrm{R}=\mathrm{La}, \mathrm{Pr}, \mathrm{Nd}$, and Eu). Phys. Rev. B 2008, 78, 134402. [CrossRef]

8. Vonsovskii, S.V.; Svirskii, M.S. ZhETF1965, 47, 1354. J. Exp. Theor. Phys. 1965, 20, 914.

9. Lyubutin, I.S.; Struzhkin, V.V.; Mironovich, A.A.; Gavriliuk, A.G.; Naumov, P.G.; Lin, J.F.; Ovchinnikov, S.G.; Sinogeikin, S.; Chow, P.; Xiao, Y.; et al. Quantum critical point and spin fluctuations in lower mantle ferropericlase. Proc. Natl. Acad. Sci. USA 2013, 110, 7142-7147. [CrossRef] [PubMed]

10. Jeong, D.W.; Choi, W.S.; Okamoto, S.; Kim, J.-Y.; Kim, K.W.; Moon, S.J.; Cho, D.-Y.; Lee, H.N.; Noh, T.W. Dimensionality control of d-orbital occupation in oxide superlattices. Sci. Rep. 2014, 4, 6124. [CrossRef] [PubMed]

11. Ivanova, N.B.; Ovchinnikov, S.G.; Korshunov, M.M.; Eremin, I.M.; Kazak, N.V. Specific features of spin, charge, and orbital ordering in cobaltites. Phys. Uspekhi 2009, 52, 789-810. [CrossRef]

12. Bernard, R.; Seikh, M. Cobalt Oxides: From Crystal Chemistry to Physics; Wiley-VCH: Weinheim, Germany, 2012.

13. Noguchi, S.; Kawamata, S.; Okuda, K.; Nojiri, H.; Motokawa, M. Evidence for the excited triplet of $\mathrm{Co}^{3+}$ in $\mathrm{LaCoO}_{3}$. Phys. Rev. B 2002, 66, 094404. [CrossRef]

14. Haverkort, M.W.; Hu, Z.; Cezar, J.C.; Burnus, T.; Hartmann, H.; Reuther, M.; Zobel, C.; Lorenz, T.; Tanaka, A.; Brookes, N.B.; et al. Spin state transition in $\mathrm{LaCoO}_{3}$ studied using soft X-ray absorption spectroscopy and magnetic circular dichroism. Phys. Rev. Lett. 2006, 97, 176405. [CrossRef] [PubMed]

15. Ropka, Z.; Radwanski, R.J. The Jahn-Teller-effect formation of the non-magnetic state of the $\mathrm{Co}^{3+}$ ion in $\mathrm{LaCoO}_{3}$. Phys. B Condens. Matter 2002, 312-313, 777-779. [CrossRef]

16. Tanabe, Y.; Sugano, S. On the absorption spectra of complex ions II. J. Phys. Soc. Jpn. 1954, 9, 766-779. [CrossRef]

17. Ovchinnikov, S.G.; Orlov, Y.S.; Nekrasov, I.A.; Pchelkina, Z.V. Electronic structure, magnetic properties, and mechanism of the insulator-metal transition in $\mathrm{LaCoO}_{3}$ taking into account strong electron correlations. J. Exp. Theor. Phys. 2011, 112, 140-151. [CrossRef]

18. Bhide, V.G.; Rajoria, D.S.; Reddy, Y.S.; Rao, G.R.; Subba Rao, G.V.; Rao, C.N.R. Localized-to-itinerant electron transitions in rare-earth cobaltates. Phys. Rev. Lett. 1972, 28, 1133-1136. [CrossRef]

19. Chang, C.Y.; Lin, B.N.; Ku, H.C.; Hsu, Y.-Y. Occurrence and variation of spin-state transitions in $\mathrm{La}_{1-x} \mathrm{Eu}_{x} \mathrm{CoO}_{3}$ cobaltates. Chin. J. Phys. 2003, 41, 662-670.

20. Thornton, G.; Morrison, F.C.; Partington, S.; Tofield, B.C.; Williams, D.E. The rare earth cobaltates: Localised or collective electron behaviour? J. Phys. C Solid State Phys. 1988, 21, 2871-2880. [CrossRef] 
21. Yan, J.-Q.; Zhou, J.-S.; Goodenough, J.B. Bond-length fluctuations and the spin-state transition in $\mathrm{LCoO}_{3}(\mathrm{~L}=$ La, Pr, and Nd). Phys. Rev. B 2004, 69, 134409. [CrossRef]

22. Ivanova, N.B.; Kazak, N.V.; Michel, C.R.; Balaev, A.D.; Ovchinnikov, S.G.; Vasil'ev, A.D.; Bulina, N.V.; Panchenko, E.B. Effect of strontium and barium doping on the magnetic state and electrical conductivity of $\mathrm{GdCoO}_{3}$. Phys. Solid State 2007, 49, 1498-1506. [CrossRef]

23. Hoch, M.J.R.; Nellutla, S.; van Tol, J.; Choi, E.S.; Lu, J.; Zheng, H.; Mitchell, J.F. Diamagnetic to paramagnetic transition in $\mathrm{LaCoO}_{3}$. Phys. Rev. B 2009, 79, 214421. [CrossRef]

24. Yamaguchi, S.; Okimoto, Y.; Tokura, Y. Bandwidth dependence of insulator-metal transitions in perovskite cobalt oxides. Phys. Rev. B 1996, 54, R11022. [CrossRef] [PubMed]

25. Asai, K.; Yoneda, A.; Yokokura, O.; Tranquada, J.M.; Shirane, G.; Kohn, K. Two spin-state transitions in $\mathrm{LaCoO}_{3}$. J. Phys. Soc. Jpn. 1998, 67, 290-296. [CrossRef]

26. Ovchinnikov, S.G.; Orlov, Y.S.; Dudnikov, V.A. Temperature and field dependent electronic structure and magnetic properties of $\mathrm{LaCoO}_{3}$ and $\mathrm{GdCoO}_{3}$. J. Magn. Magn. Mater. 2012, 324, 3584-3587. [CrossRef]

27. Jirák, Z.; Hejtmanek, J.; Knižek, K.; Novak, P.; Šantava, E.; Fujishiro, H. Magnetism of perovskite cobaltites with Kramers rare-earth ions. J. Appl. Phys. 2014, 115, 17E118. [CrossRef]

28. Tachibana, M.; Yoshida, T.; Kawaji, H.; Atake, T.; Takayama-Muromachi, E. Evolution of electronic states in $\mathrm{RCoO}_{3}(\mathrm{R}=$ rare earth): Heat capacity measurements. Phys. Rev. B 2008, 77, 094402. [CrossRef]

29. Dudnikov, V.A.; Orlov, Y.S.; Gavrilkin, S.Y.; Gorev, M.V.; Vereshchagin, S.N.; Solovyov, L.A.; Perov, N.S.; Ovchinnikov, S.G. Effect of $\mathrm{Gd}$ and $\mathrm{Sr}$ ordering in A sites of doped $\mathrm{Gd}_{0.2} \mathrm{Sr}_{0.8} \mathrm{CoO}_{3-\delta}$ perovskite on its structural, magnetic, and thermodynamic properties. J. Phys. Chem. C 2016, 120, 13443-13449. [CrossRef]

30. Taguchi, H. Electrical properties and spin state of the $\mathrm{Co}^{3+}$ ion in $\left(\mathrm{Nd}_{1-x} \mathrm{Gd}_{x}\right) \mathrm{CoO}_{3}$. Phys. B Condens. Matter 2002, 311, 298-304. [CrossRef]

31. Yu, J.; Phelan, D.; Louca, D. Spin-state transitions in $\mathrm{PrCoO}_{3}$ investigated by neutron scattering. Phys. Rev. $B$ 2011, 84, 132410. [CrossRef]

32. Umemoto, K.; Seto, Y.; Masuda, Y. Structure and magnetic property of $\mathrm{Ce}_{x} \mathrm{Eu}_{1-x} \mathrm{CoO}_{3}$ prepared by means of the thermal decomposition of $\mathrm{Ce}_{x} \mathrm{Eu}_{1-x}\left[\mathrm{Co}(\mathrm{CN})_{6}\right] \cdot n \mathrm{H}_{2} \mathrm{O}$. Thermochimicaacta 2005, 431, 117-122. [CrossRef]

33. Brinks, H.W.; Fjellvasg, H.; Kjekshus, A.; Hauback, B.C. Structure and magnetism of $\operatorname{Pr}_{1-x} \operatorname{Sr}_{x} \mathrm{CoO}_{3-\delta} . J$. Solid State Chem. 1999, 147, 464-477. [CrossRef]

34. Spinicci, R.; Faticanti, M.; Marini, P.; De Rossi, S.; Porta, P. Catalytic activity of $\mathrm{LaMnO}_{3}$ and $\mathrm{LaCoO}_{3}$ perovskites towards VOCs combustion. J. Mol. Catal. A Chem. 2003, 197, 147-155. [CrossRef]

35. Takeda, Y.; Ueno, H.; Imanishi, N.; Yamamoto, O.; Sammes, N.; Phillipps, M.B. $\mathrm{Gd}_{1-x} \mathrm{Sr}_{x} \mathrm{CoO}_{3}$ for the electrode of solid oxide fuel cells. Solid State Ion. 1996, 86, 1187-1190. [CrossRef]

36. Liu, H.; Wu, Y.P.; Rahm, E.; Holze, R.; Wu, H.Q. Cathode materials for lithium ion batteries prepared by sol-gel methods. J. Solid State Electrochem. 2004, 8, 450-466. [CrossRef]

37. Teraoka, Y.; Zhang, H.M.; Okamoto, K.; Yamazoe, N. Mixed ionic-electronic conductivity of $\mathrm{La}_{1-x} \mathrm{Sr}_{x} \mathrm{Co}_{1-y} \mathrm{Fe}_{y} \mathrm{O}_{3-\delta}$ perovskite-type oxides. Mater. Res. Bull. 1988, 23, 51-58. [CrossRef]

38. Michel, C.R.; Gago, A.S.; Guzmán-Colín, H.; López-Mena, E.R.; Lardizábal, D.; Buassi-Monroy, O.S. Electrical properties of the perovskite $\mathrm{Y}_{0.9} \mathrm{Sr}_{0.1} \mathrm{CoO}_{3-\delta}$ prepared by a solution method. Mater. Res. Bull. 2004, 39, 2295-2302. [CrossRef]

39. Orlov, Y.S.; Solovyov, L.A.; Dudnikov, V.A.; Fedorov, A.S.; Kuzubov, A.A.; Kazak, N.V.; Voronov, V.N.; Vereshchagin, S.N.; Shishkina, N.N.; Perov, N.S.; et al. Structural properties and high-temperature spin and electronic transitions in $\mathrm{GdCoO}_{3}$ : Experiment and theory. Phys. Rev. B 2013, 88, 235105. [CrossRef]

40. Solovyov, L.A. Full-profile refinement by derivative difference minimization. J. Appl. Crystallogr. 2004, 37, 743-749. [CrossRef]

41. Kharko, O.V.; Vasylechko, L.O.; Ubizskii, S.B.; Pashuk, A.; Prots, Y. Structural behavior of continuous solid solution $\mathrm{SmCo}_{1-x} \mathrm{Fe}_{x} \mathrm{O}_{3}$. Funct. Mater. 2014, 21, 226-232. [CrossRef]

42. Knížek, K.; Hejtmánek, J.; Jirák, Z.; Tomeš, P.; Henry, P.; André, G. Neutron diffraction and heat capacity studies of $\mathrm{PrCoO}_{3}$ and $\mathrm{NdCoO}_{3}$. Phys. Rev. B 2009, 79, 134103. [CrossRef]

43. Orlov, Y.S.; Dudnikov, V.A.; Gorev, M.V.; Vereshchagin, S.N.; Solov'ev, L.A.; Ovchinnikov, S.G. Thermal properties of rare earth cobalt oxides and of $\mathrm{La}_{1-x} \mathrm{Gd}_{x} \mathrm{CoO}_{3}$ solid solutions. JETP Lett. 2016, 103, 607-612. [CrossRef]

44. Seijas, J.G.; Prado-Gonjal, J.; Brande, D.A.; Terry, I.; Moran, E.; Schmidt, R. Microwave-assisted synthesis, microstructure, and magnetic properties of rare-earth cobaltites. Inorg. Chem. 2016, 56, 627-633. [CrossRef] 
45. Panfilov, A.S.; Grechnev, G.E.; Lyogenkaya, A.A.; Pashchenko, V.A.; Zhuravleva, I.P.; Vasylechko, L.O.; Hreb, V.M.; Turchenko, V.A.; Novoselov, D. Magnetic properties of $\mathrm{RCoO}_{3}$ cobaltites $(\mathrm{R}=\mathrm{La}, \mathrm{Pr}, \mathrm{Nd}, \mathrm{Sm}$, Eu). Effects of hydrostatic and chemical pressure. Phys. B Condens. Matter 2019, 553, 80-87. [CrossRef]

46. Vonsovsky, S.V. Magnetism; Nauka: Moscow, Russia, 1971; p. 1032.

47. Ivanova, N.B.; Kazak, N.V.; Michel, C.R.; Balaev, A.D.; Ovchinnikov, S.G. Low-temperature magnetic behavior of the rare-earth cobaltites $\mathrm{GdCoO}_{3}$ and $\mathrm{SmCoO}_{3}$. Phys. Solid State 2007, 49, 2126-2131. [CrossRef]

48. Ropka, Z.; Radwanski, R.J. ${ }^{5}$ D term origin of the excited triplet in $\mathrm{LaCoO}_{3}$. Phys. Rev. B 2003, 67, 172401. [CrossRef]

49. Zvezdin, A.K.; Matveev, V.M.; Mukhin, A.A.; Popov, A.I. Rare Earth Ions in Magnetically Ordered Crystals; Moscow Izdatel Nauka: Moscow, Russia, 1985. (In Russian)

50. VanVleck, J.H. The Theory of Electronic and Magnetic Susceptibilities; Oxford University Press: Oxford, UK, 1932.

51. Mott, N.F.; Davis, E.A. Electronic Processes in Non Crystalline Materials; Clarendon Press: Oxford, UK, 1971; p. 437.

52. Berggold, K.; Kriener, M.; Zobel, C.; Reichl, A.; Reuther, M.; Müller, R.; Freimuth, A.; Lorenz, T. Thermal conductivity, thermopower, and figure of merit of $\mathrm{La}_{1-x} \mathrm{Sr}_{x} \mathrm{CoO}_{3}$. Phys. Rev. B 2005, 72, 155116. [CrossRef]

53. Scherrer, B.; Harvey, A.S.; Tanasescu, S.; Teodorescu, F.; Botea, A.; Conder, K.; Grundy, A.N.; Martynczuk, J.; Gauckler, L.J. Correlation between electrical properties and thermodynamic stability of $\mathrm{A} \mathrm{CoO}_{3-\delta}$ perovskites (A = La, Pr, Nd, Sm, Gd). Phys. Rev. B 2011, 84, 085113. [CrossRef]

54. Nagaev, E.L.; Podel'shchikov, A.I. Phase separation and resistivity jumps in Co compounds and other materials with low-spin-high-spin transitions. J. Phys. Condens. Matter 1996, 8, 5611-5620. [CrossRef]

55. Giblin, S.R.; Terry, I.; Clark, S.J.; Prokscha, T.; Prabhakaran, D.; Boothroyd, A.T.; Wu, J.; Leighton, C. Observation of magnetic excitons in $\mathrm{LaCoO}_{3}$. Europhys. Lett. 2005, 70, 677-683. [CrossRef]

56. Vasil'chikova, T.N.; Kuz'mova, T.G.; Kamenev, A.A.; Kaul', A.R.; Vasil'ev, A.N. Spin states of cobalt and the thermodynamics of $\mathrm{Sm}_{1-\mathrm{x}} \mathrm{Ca}_{\mathrm{x}} \mathrm{CoO}_{3-\delta}$ solid solutions. JETP Lett. 2013, 97, 34-37. [CrossRef]

Sample Availability: Samples of the compounds are not available from the authors.

(C) 2020 by the authors. Licensee MDPI, Basel, Switzerland. This article is an open access article distributed under the terms and conditions of the Creative Commons Attribution (CC BY) license (http://creativecommons.org/licenses/by/4.0/). 